\title{
AA Tauri's sudden and long-lasting deepening: enhanced extinction by its circumstellar disk ${ }^{\star}$
}

\author{
J. Bouvier ${ }^{1}$, K. Grankin ${ }^{2}$, L. E. Ellerbroek ${ }^{3}$, H. Bouy ${ }^{4}$, and D. Barrado ${ }^{4}$ \\ ${ }^{1}$ UJF-Grenoble 1/CNRS-INSU, Institut de Planétologie et d'Astrophysique de Grenoble (IPAG) UMR 5274, 38041 Grenoble, France \\ e-mail: Jerome.Bouvier@obs.ujf-grenoble.fr \\ 2 Crimean Astrophysical Observatory, Scientific Research institute, 98409 Nauchny, Crimea, Ukraine \\ 3 Astronomical Institute Anton Pannekoek, Science Park 904, PO Box 94249, 1090 GE Amsterdam, The Netherlands \\ ${ }^{4}$ Centro de Astrobiologa (INTA-CSIC), PO Box 78, 28691 Villanueva de la Cañada, Spain
}

Received 1 March 2013 / Accepted 25 March 2013

\begin{abstract}
Context. AA Tau has been monitored for more than 20 years since 1987 and exhibited a nearly constant brightness level of $V=$ $12.5 \mathrm{mag}$. We report here that in 2011 it suddenly faded, becoming $2 \mathrm{mag}$ fainter in the $V$-band, and has remained in this deep state since then.

Aims. We investigate the origin of the sudden dimming of the AA Tau system.

Methods. We report on new optical and near-IR photometry and spectroscopy obtained during the fading event.

Results. The system appears to be much redder and fainter than it was in the bright state. Moreover, the $8.2 \mathrm{~d}$ photometric period continuously observed for more than 20 years is not detected during most of the deep state. The analysis of the system's brightness and colors suggests that the visual extinction on the line of sight has increased by about 3-4 mag in the deep state. At optical wavelengths, the system appears to be dominated by scattered light, probably originating from the upper surface layers of a highly inclined circumstellar disk. The profiles of the Balmer lines have significantly changed as well, with the disappearance of a central absorption component regularly observed in the bright state. We ascribe this change to the scattering of the system's spectrum by circumstellar dust. Remarkably, the mass accretion rate in the inner disk and onto the central star has not changed as the system faded.

Conclusions. We conclude that the deepening of the AA Tau system is due to a sudden increase of circumstellar dust extinction on the line of sight without concomitant change in the accretion rate. We suggest that the enhanced obscuration may be produced by a nonaxisymmetric overdense region in the disk, located at a distance of $7.7 \mathrm{AU}$ or more, that was recently brought into the line of sight by its Keplerian motion around the central star.
\end{abstract}

Key words. accretion, accretion disks - stars: individual: AA Tau - stars: pre-main sequence - stars: variables: T Tauri, Herbig Ae/Be

\section{Introduction}

A young, low-mass member of the 1-3 Myr Taurus association (Kenyon \& Hartmann 1995), AA Tau has provided many clues to the magnetically mediated accretion process occurring in young stellar objects. The strong large-scale magnetic fields measured at the surface of young low-mass stars (Donati \& Landstreet 2009) are thought to be able to disrupt the inner accretion disk surrounding the central star up to a distance of several stellar radii (Camenzind 1990; Königl 1991). From the inner disk edge to the stellar surface, magnetically controlled accretion funnel flows develop, eventually reaching the stellar surface at nearly free-fall velocity, thus creating a localized accretion shock seen as a hot spot (e.g., Bertout et al. 1988). In addition, the magnetic star-disk interaction is expected to create a nonaxisymmetric disturbance at the inner disk edge, i.e., an inner disk warp, in the

\footnotetext{
* Based on observations made with telescopes at the Crimean Astrophysical Observatory, at the Calar Alto Observatory, and at ESO Paranal Observatory under programme ID 288.C-5026(A).

$\star \star$ Photometric measurements are only available at the CDS via anonymous ftp to cdsarc.u-strasbg. fr (130.79.128.5) or via http://cdsarc.u-strasbg.fr/viz-bin/qcat?J/A+A/557/A77
}

case of an inclined magnetosphere (e.g. Terquem \& Papaloizou 2000). Many clues have been gathered that suggest that magnetospheric accretion is quite a common process indeed in young accreting low-mass stars (see, e.g., Bouvier et al. 2007a for a review).

Thanks to its close to edge-on orientation on the line of sight ( $i \simeq 70-75^{\circ}$, Bouvier et al. 2003; O'Sullivan et al. 2005; Cox et al. 2013), AA Tau has yielded deep insight into the actual geometry and dynamics of the magnetospheric accretion process. Spectrophotometric monitoring campaigns performed on this source (cf. Bouvier et al. 1999, 2003, 2007) and polarimetric studies (Ménard et al. 2003; O'Sullivan et al. 2005) have investigated the geometry of the magnetically controlled accretion flows from the inner disk to the stellar surface, as well as the connection between the funnel flows and the hot spots at the stellar surface. They have also revealed a nonaxisymmetric inner disk warp, presumably driven by the interaction of the disk with an inclined dipole, and have highlighted the very dynamical nature of this interaction, with significant structural changes occurring on a timescale of weeks. Direct magnetic field measurements have confirmed an inclined 2-3 kG magnetic dipole at the surface of AA Tau (Donati et al. 2010), and the circumstellar disk has been 
recently directly imaged, confirming its warped structure (Cox et al. 2013).

While AA Tau has served as a Rosetta Stone for the understanding of the geometry and dynamics of the accretion flow in young magnetic stars, many other cases have now been reported that display similar photometric variations and associated spectroscopic signatures. Indeed, a distinctive signature of AA Tau-like light curves is the occurrence of quasi-periodic dips that interrupt a nearly constant bright photometric level. The dips occur on a week's timescale and are interpreted as resulting from the partial occultation of the central star by the warped inner disk edge as it rotates at Keplerian velocity at a distance of a few stellar radii from the stellar surface (Bouvier et al. 1999). In a large-scale CoRoT-based photometric study of the $3 \mathrm{Myr}$ NGC 2264 star-forming region, Alencar et al. (2010) reported a fraction of $40 \pm 10 \%$ of AA Tau-like light curves among classical T Tauri stars with optically thick accretion disks. Hence, the AA Tau phenomenon, i.e., the quasi-periodic obscuration of the central star by a nonaxisymmetric inner disk, is found to be fairly common, as long as a proper sampling of the photometric variations is secured (see also Artemenko et al. 2012; Cody et al. 2013).

AA Tau itself has remained at a nearly constant maximum brightness level ( $V \simeq 12.4-12.6 \mathrm{mag}$ ) between 1987 and 2010 , i.e., for at least 24 years (Grankin et al. 2007). We report here that it suddenly lost about 2 mag in 2011 and has remained in this deep state until now (March 2013). We present here photometric and spectroscopic observations obtained during the deep state of the system. In Sect. 2, we detail the photometric and spectroscopic observations. In Sect. 3, we present the photometric and spectroscopic properties of the system in the deep state. In Sect. 4, we show that the current state of the AA Tau system can be consistently interpreted as resulting from a sudden increase of extinction on the line of sight and discuss its possible origin in the circumstellar disk.

\section{Observations}

\subsection{Photometry}

Optical observations of AA Tau were obtained at the Crimean Astrophysical Observatory (CrAO) from October 2007 to February 2013. The measurements were carried out with the CrAO $1.25 \mathrm{~m}$ telescope (AZT-11) equipped with either the Finnish five-channel photometer or the FLI 1001E CCD detector. The photomultiplier tube was used during the bright phases of the star $(V \leq 14 \mathrm{mag}$ ) and the CCD detector for the fainter phases. All photometric observations were obtained in the $V$ and $R_{J}$ filters. Differential photometry was performed on CCD images and absolute photometry from photomultiplier observations, with an accuracy of about $0.05 \mathrm{mag}$ in both filters. The two comparison stars are located less than 4 arcmin away from AA Tau (these are J04344511+2424432 and $\mathrm{J} 04344826+2428532$ in the 2 MASS catalog). All data reduction procedures followed those previously described in Bouvier et al. (2003).

Additional $B V R_{\mathrm{c}} I_{\mathrm{c}}$ photometry was obtained on December 23, 2011 using the Cafos focal reducer (Meisenheimer 1994) in direct-imaging mode with CCD SITE1d_15 on the $2.2 \mathrm{~m}$ Calar Alto Telescope. Individual BVRI images were bias subtracted and flat-field corrected. Differential photometry was performed between AA Tau and the same comparison stars as at CrAO. The resulting photometry is accurate to about $0.05 \mathrm{mag}$.
Table 1. CAHA optical and near-IR photometry of the system.

\begin{tabular}{lcccc}
\hline \hline JD 2 400000 & $V$ & $B$ & $R_{\mathrm{c}}$ & $I_{\mathrm{c}}$ \\
55919.5426 & 15.578 & 17.145 & 14.351 & 12.943 \\
\hline JD 2400000 & $J$ & $H$ & $K$ & \\
55909.3290 & 10.464 & 9.202 & 8.458 & \\
55913.4358 & 10.614 & 9.266 & 8.431 & \\
55914.6103 & 10.525 & 9.210 & 8.446 & \\
\hline
\end{tabular}

Near-infrared (near-IR) $J H K$-photometry was obtained on December 13, 17, and 18, 2011 with the Omega2000 camera (Kovacs et al. 2004) mounted on the $3.5 \mathrm{~m}$ Calar Alto telescope. The $\mathrm{O} 2000$ individual images were first processed using the Alambic EIS-MVM pipeline (Vandame 2002) adapted to the characteristics of the instrument. Briefly, the pipeline includes standard pre-processing procedures (overscan and bias subtraction, flat-fielding), followed by an unbiased two-pass background subtraction, registration, and stacking of the individual images. A second-order astrometric solution was derived using the 2MASS catalog as reference. Differential photometry was obtained with the same neighboring comparison stars, whose $J H K$ photometry was obtained from the 2MASS catalog.

The full set of photometric measurements obtained at $\mathrm{CrAO}$ from 2007 to 2013 is available at the CDS. The additional photometry obtained at Calar Alto Observatory is listed in Table 1.

\subsection{Spectroscopy}

Low-resolution $(\sim 0.7 \mathrm{~nm})$ optical spectroscopy was performed on 13 and 23 December 2011 using the Cafos focal reducer (Meisenheimer 1994) on the $2.2 \mathrm{~m}$ Calar Alto telescope equipped with the g-100 grism. The exposure time was $1800 \mathrm{~s}$ for each spectrum, and the slit width was $11^{\prime \prime} 5$. Two $1800 \mathrm{~s} \mathrm{spec-}$ tra were obtained in sequence on Dec. 13, 2011, and were subsequently averaged.The spectrophotometric standard G191B2b was observed at the same airmass as AA Tau on both nights. After the 2D images were bias corrected and flat-fielded, the object's and neighboring sky's spectra were extracted using the IRAF/twodspec package. The wavelength was calibrated using the $\mathrm{HgHeRb}$ spectral lamp. Owing to the low number of lines available in the arc spectrum over the 480-770 $\mathrm{nm}$ range, the wavelength calibration is not accurate to better than $2 \mathrm{~nm}$. Finally, the response of the instrument was corrected for using the spectrum of the spectrophotometric standard G191B2b.

Medium-resolution spectroscopy was obtained with ESO VLT/XSHOOTER on 25 February 2012. XSHOOTER is a cross-dispersed echelle spectrograph with three arms: UVB (300-590 nm), VIS (550-1020 nm) and near-IR (1000-2480 nm) (D'odorico et al. 2006; Vernet et al. 2011). Slit widths of 1. .' $3,0.7$ and 0.4 were chosen in these arms to deliver a corresponding spectral resolution of 4000, 11000 , and 11300 in the respective wavelength domains. The standard steps of data reduction (order extraction, flat-fielding, wavelength calibration) were applied using the XSHOOTER pipeline (Modigliani et al. 2010). The observations comprised four exposures at alternating offsets along the slit (nodding), allowing for accurate sky subtraction, with a total exposure time of $1000 \mathrm{~s}$. The resulting one-dimensional spectra were flux-calibrated using a spectrum of the spectrophotometric standard star LTT3218, which was taken directly before AA Tau. We corrected for slitlosses, taking into account the average seeing $\left(1{ }^{\prime \prime} 0\right.$ in $\left.V\right)$, resulting in a scaling up of $1.24,1.93$ and 3.21 in the $U V B$, VIS and near-IR arms, respectively. The resulting flux calibration agrees well in the overlapping wavelength regions between the arms. 
J. Bouvier et al.: AA Tauri's sudden and long-lasting deepening: enhanced extinction by its circumstellar disk

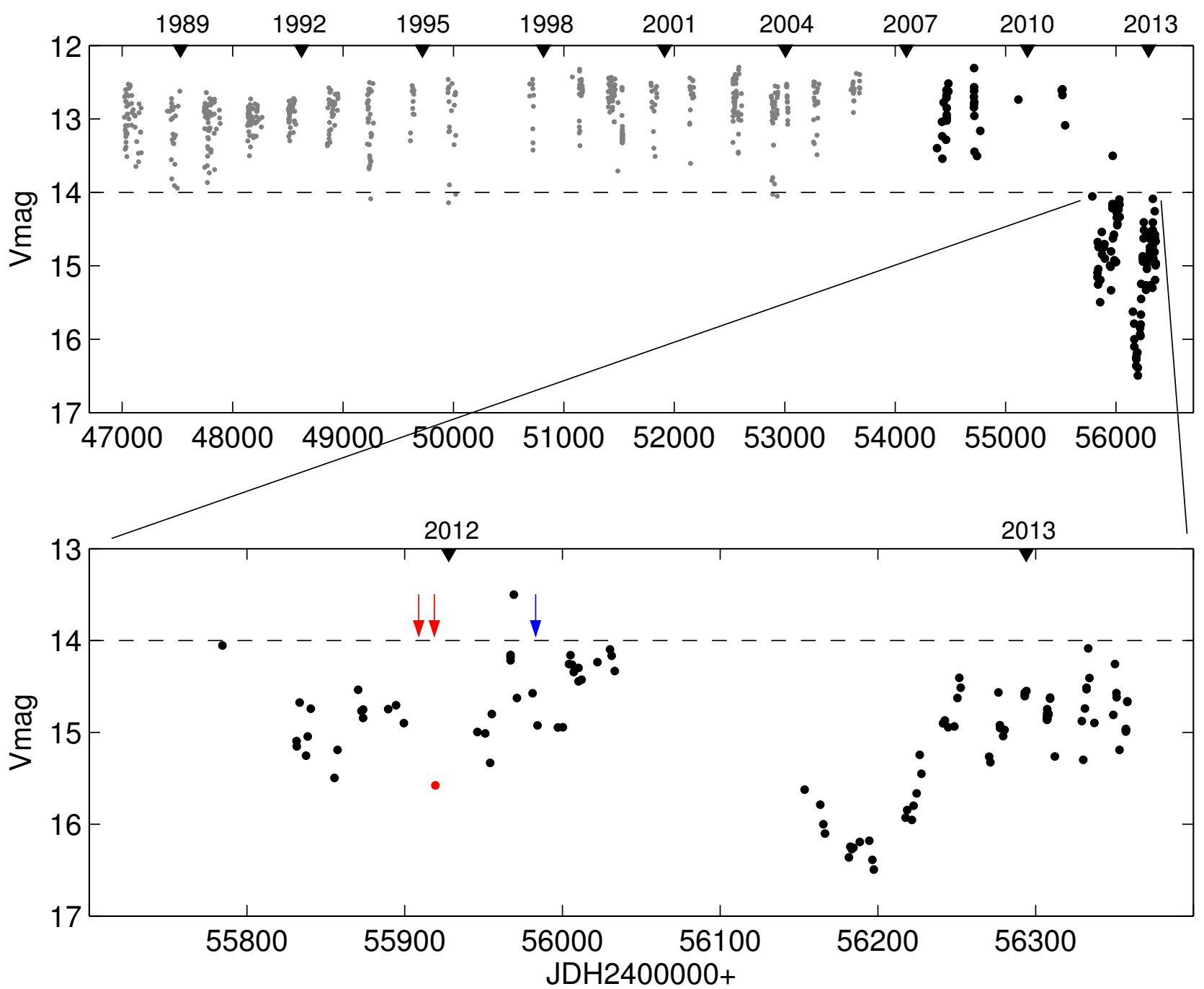

Fig. 1. $V$-band AA Tau light-curve spanning 26 years is shown on the top panel. Gray circles refer to Mt Maidanak measurements (Grankin et al. 2007) while dark circles are new measurements from CrAO. The $V=14$ mag brightness level is shown as dashed line for reference. The bottom panel shows a zoom on the two last observing seasons (2011-2012). The two leftmost red arrows in late-2011 are the time the Calar Alto lowresolution spectra were obtained, and the red photometric point below the second arrow is the Calar Alto photometric measurement. The rightmost blue arrow in early 2012 corresponds to the time the ESO/XSHOOTER spectrum was obtained.

\section{Results}

\subsection{Photometric variability}

The long-term light curve of AA Tau in the V filter recorded over 26 years is shown in Fig. 1. From 1987 till the beginning of 2011, the system was almost never fainter than $V=14$ mag, even in the center of eclipses. During the 20 seasons over this time span for which we gathered enough data, the maximum brightness level smoothly varied within the range $12.3-12.7 \mathrm{mag}$, the average brightness level remained between 12.7 and $13.1 \mathrm{mag}$, and the seasonal photometric amplitude varied between 0.6 and $1.7 \mathrm{mag}$ in the $V$ band (cf. Grankin et al. 2007). Periodic light variations are present over this time span with a period of $8.19 \mathrm{~d}$ (Artemenko et al. 2012). However, at some point in 2011 the average brightness level suddenly decreased by $\simeq 2 \mathrm{mag}$, from $V=$ 12.8 to $V=15$, and the maximum brightness level dimmed below $V=14$.

An enlargement of the 2011-2013 seasons is shown in Fig. 1. The deep minimum has continued for more than 500 days. The lowest brightness level ( $V \simeq 16.5 \mathrm{mag}$ ) was registered at the end of September 2012. From 20 November 2012 on (JD $\geq 56251$ ), the average brightness level apparently remained roughly constant at $V \simeq 14.8$, and the photometric amplitude reached up to 0.9 mag. Both mid-term change, on a timescale of several weeks (e.g. JD 56 150-JD 56250), and short-term variations, up to 0.4-0.6 mag per day, are recorded. In particular, an UX Ori-like smooth and long minimum, with little photometric dispersion, is quite noticeable in late 2012. A search for periodicities in the 2011/12 and 2012/13 photometric datasets, analyzed either separately or combined, did not reveal any significant periodic signal. To investigate the existence of the $8.19 \mathrm{~d}$ period that the system exhibited over more than 20 years in the bright state (Grankin et al. 2007; Artemenko et al. 2012), we nevertheless examined phase diagrams for various parts of the deep-state light curve. The results are shown in Fig 2. Neither the 2011/12 season nor the first half of the 2012/13 season reveal any sign of coherent photometric variations on a $8.19 \mathrm{~d}$ timescale. However, surprisingly enough, the phase diagram for the second half of the 2012/13 season (JD 56250-56358) strongly suggests that the $8.19 \mathrm{~d}$ period is recovered over this specific $\sim 3$ month time interval with a $V$-band amplitude of about $0.7 \mathrm{mag}$. 

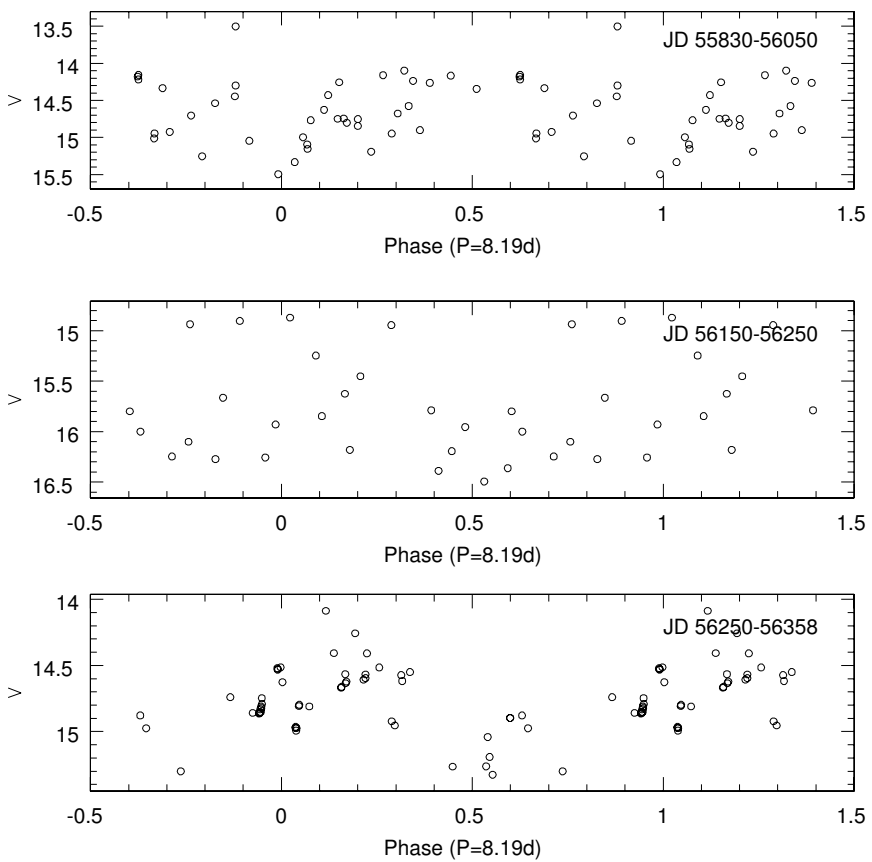

Fig. 2. AA Tau's deep state light curve folded in phase assuming a period of 8.19d. Top panel: 2011/12 season (JD 55 830-56 050). Middle panel: first half of the 2012/13 season (JD 56 150-56250); Bottom panel: second half of the 2012/13 season (JD 56 250-56358). The origin of the photometric phase is arbritrary.

The $\left(V, V-R_{j}\right)$ color behavior of the system is shown in Fig. 3 over the full 26-year time span of AA Tau monitoring. As long as the star remained brighter than $V=15$, the color variations of the system are nearly parallel to, though slightly bluer than, the reddening slope expected for small ISM-type grains. In contrast, the system does not redden more when it becomes fainter than $V=14.5 \mathrm{mag}$. Instead, the full range of $\left(V-R_{j}\right)$ colors from 1.5 to $1.8 \mathrm{mag}$ is observed between $V=15$ and $V=16.5 \mathrm{mag}$. This blueing effect is typical of UX Ori type variables (Grinin et al. 1991; Herbst et al. 1994), whose photometric behavior is caused by variable circumstellar extinction. It is usually interpreted as scattered light becoming a major component of the system's flux as direct light from the central object is depressed.

Figure 3 also shows the near-infrared $(J-H, H-K)$ colorcolor diagram of the system as it switches from the bright to the deep state. In the bright state, AA Tau is located close to the $\mathrm{T}$ Tauri locus, indicative of moderate accretion and little extinction, in agreement with previous estimates (e.g. Bouvier et al. 1999). In the deep photometric state, the near-IR colors of the system are much redder. The transition between the bright and deep states in this diagram suggests a 4 mag increase of the visual extinction on the line of sight. As discussed below, since the system is seen nearly edge-on, the sudden increase of extinction on the line of sight is probably of circumstellar origin.

\subsection{Spectroscopy}

The low-resolution AA Tau spectra obtained on Dec. 13 and 23, 2011, are shown in Fig. 4 over the wavelength range 480-770 $\mathrm{nm}$. A clear photospheric late-type spectrum is seen as well as a few emission lines including $\mathrm{H} \alpha, \mathrm{H} \beta$, HeI $587.6 \mathrm{~nm}$, and the [OI] 630.0/636.4 nm doublet. The two spectra obtained during the deep state are compared with the
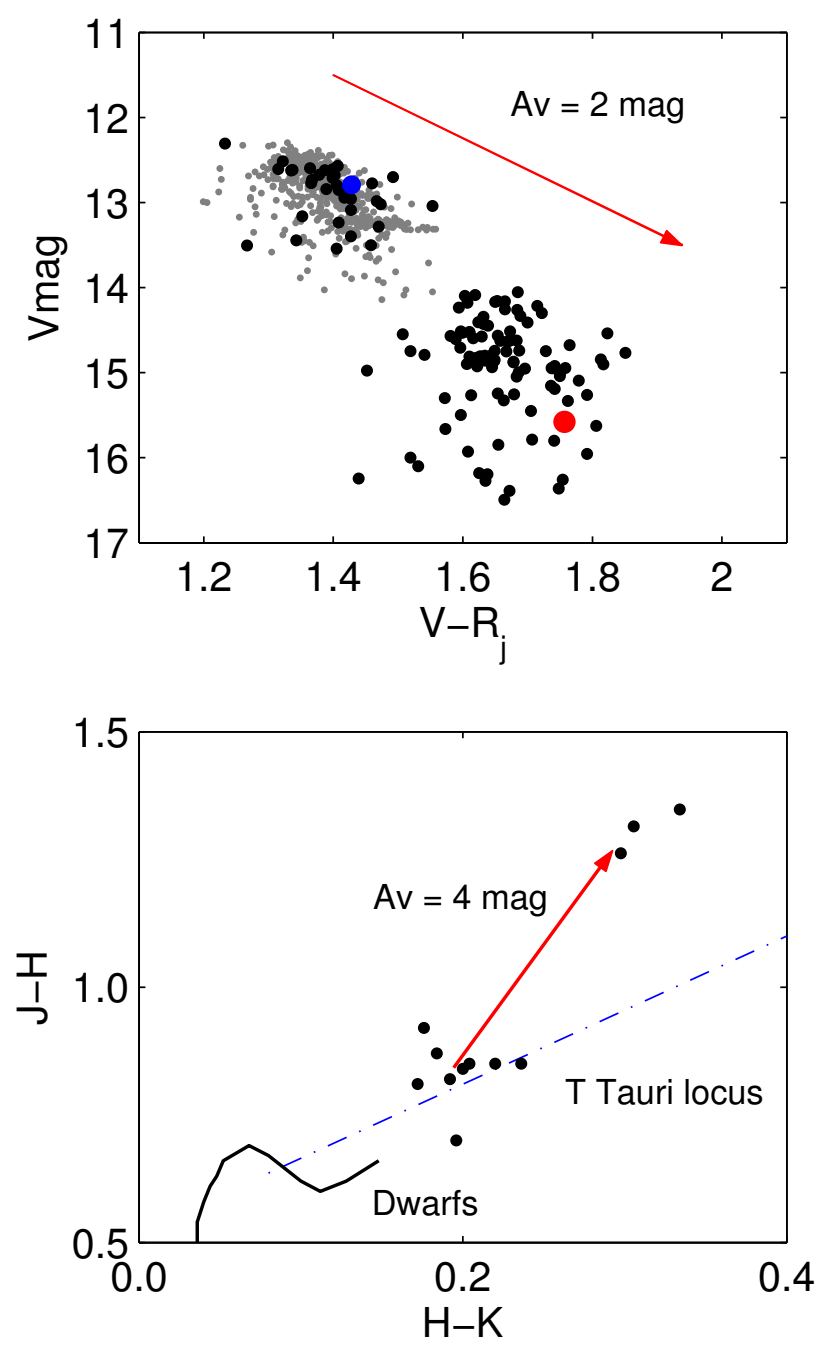

Fig. 3. AA Tau's color behavior. Top panel: $\left(V, V-R_{J}\right)$ color-magnitude diagram. The symbols are the same as in Fig. 1. The arrow indicates the color slope expected for $A_{V}=2 \mathrm{mag}$ of interstellar extinction. The large blue circle illustrates the bright state of the system, as recorded on 19 Nov. 1995 (from Bouvier et al. 1999). The large red circle corresponds to the deep-state photometry obtained on 23 Dec. 2011. The system becomes bluer at the faintest brightness level. Bottom panel: $(J-H$, $H-K$ ) color diagram. The black curve indicates the dwarf locus, and the blue dashed-dotted curve the T Tauri locus. The arrow indicates the color slope expected for $A_{V}=4$ mag of ISM-like extinction. JHK photometry for the bright state is taken from Bouvier et al. (1999) and for the faint state from Calar Alto measurements listed in Table 1.

average of 57 low-resolution spectra obtained in Nov. 1995, i.e., when AA Tau was still in a bright state (cf. Bouvier et al. 1999). The photospheric features have not changed significantly between the two epochs, which suggests that AA Tau has retained a spectral type of K7 in the deep state. A major difference, however, is the continuum slope, which is much steeper in the 2011 spectra than in those taken in the bright state, which indicates increased reddening. Assuming a standard interstellar extinction law $\left(R_{V}=3.1\right.$, Cardelli et al. 1989), we attempted to reproduce the continuum slopes of the 2011 spectra by reddening the 1995 bright state spectrum. We obtained good fits to the 2011 Dec. 13 and 23 continuum spectral slopes by adding 2.4 mag and $1.1 \mathrm{mag}$ of visual extinction to the 1995 spectrum, respectively. The system had a $V$-band magnitude of $V=15.58$ at the time the Dec. 23 spectrum was obtained, i.e., about 2.8 mag 

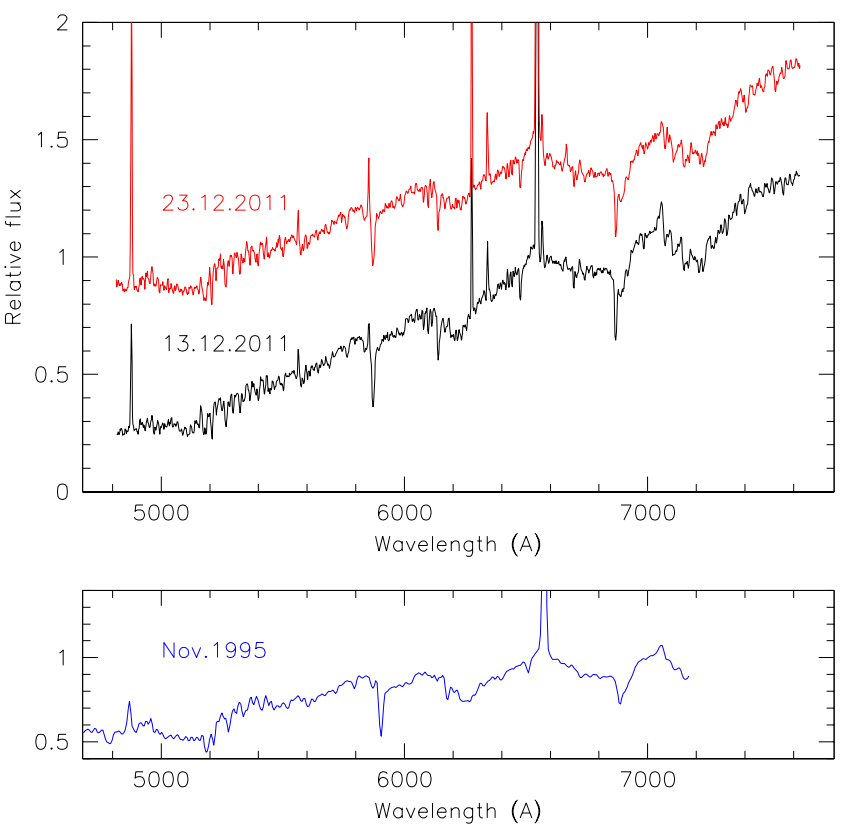

Fig. 4. Low-resolution optical spectra of the AA Tau system. Top panel: the Calar Alto Cafos spectra obtained on Dec. 13 and 23 Dec. 2011 are shown over the wavelength range $480-770 \mathrm{~nm}$. The continuum has been normalized to unity at the base of the $\mathrm{H} \alpha$ line and the flux of the latter spectrum shifted by +0.5 for clarity. Lower panel: the average of 57 AA Tau lowres spectra obtained in the bright state in Nov. 1995 is shown for comparison (from Bouvier et al. 1999).

Table 2. Equivalent width $(\AA)$ of emission lines in CAHA spectra.

\begin{tabular}{lcccccc}
\hline \hline Line & $\mathrm{H} \alpha$ & $\mathrm{H} \beta$ & $\mathrm{HeI}$ & {$[\mathrm{OI}]$} & {$[\mathrm{OI}]$} & $\mathrm{LiI}$ \\
$\lambda(\mathrm{nm})$ & 656.3 & 486.1 & 587.6 & 630.0 & 636.4 & 670.7 \\
\hline 13.12 .2011 & 31.4 & 10. & 0.8 & 5.8 & 1.5 & -0.49 \\
23.12 .2011 & 70. & 19. & 1.6 & 7.5 & 2.1 & -0.36 \\
\hline
\end{tabular}

fainter than AA Tau's bright state. Yet, the spectral slope suggests an additional extinction amounting to only 1.1 mag compared with the bright state. This confirms that the system becomes bluer when it is fainter, as reported from the photometry above. A similar behavior was already observed in the changing continuum slope of the 1995 spectral series (cf. Bouvier et al. 1999) and was ascribed to the increasing contribution of scattered light as the system dims.

Another clear difference between the 1995 and 2011 spectra are the additional emission lines in the latter. While the $\mathrm{H} \alpha$ and $\mathrm{H} \beta$ lines were already observed in emission in 1995, the new spectra additionally exhibit clear emission in the HeI 587.6 and [OI] 630.0/636.4 $\mathrm{nm}$ lines ${ }^{1}$. The emission line equivalent widths were measured on both 2011 spectra by Gaussian fitting to the line profiles. The results are listed in Table 2. Clearly, the strength of the Balmer and HeI lines in the Dec. 23 spectrum is twice as large as it was in the Dec. 13 one. Whether this reflects actual line flux variation or merely a variable continuum level, we cannot tell because we do not have simultaneous photometry for the latter spectrum. However, we notice that the equivalent width of the [OI]630.0/636.4 nm lines also increased by a factor of about 1.4 between the two spectra. The [OI] lines are

\footnotetext{
1 We disregard the weak [OI]557.7 nm emission as it is significantly polluted by sky brightness and could partly result from unperfect sky subtraction.
}

thought to form far from the star, presumably in the large-scale jet recently imaged by HST (Cox et al. 2013), and are therefore not expected to intrinsically vary on such a short timescale (cf. Bouvier et al. 1999). This suggests that the continuum level of the Dec. 23 spectrum was indeed about a factor of 1.4 lower than that of the Dec. 13 spectrum. The dimming of the continuum may thus at least partly account for the enhanced equivalent width of the Balmer and HeI lines in the Dec. 23 spectrum, whose slope is also bluer than that of the Dec. 13 spectrum.

Combining the measured equivalent widths with the photometric measurement obtained simultaneously with the Dec. 23 spectrum, we derived the $\mathrm{H}_{\alpha}$ and $\mathrm{H}_{\beta}$ emission line flux. The observed line flux is given by $F\left(\mathrm{H}_{\alpha}\right) \simeq F_{R}^{0} \times E W\left(\mathrm{H}_{\alpha}\right) \times 10^{\left(-0.4 \cdot m_{R}\right)}$ and $F\left(\mathrm{H}_{\beta}\right) \simeq F_{B}^{0} \times E W\left(\mathrm{H}_{\beta}\right) \times 10^{\left(-0.4 \cdot m_{B}\right)}$, where $m_{R}$ and $m_{B}$ are the $V$ - and $B$ magnitudes of the system, while $F_{B}^{0}=1880 \mathrm{Jy}$ and $F_{R}^{0}=3080 \mathrm{Jy}$ are the fluxes for a zero magnitude star in the $B$ - and $V$ band, respectively. This yields $F\left(\mathrm{H}_{\alpha}\right)=2.9 \times 10^{-13}$ and $F\left(\mathrm{H}_{\beta}\right)=1.1 \times 10^{-14} \mathrm{erg} \mathrm{cm}^{2} \mathrm{~s}^{-1}$. For a distance of $140 \mathrm{pc}$, the line fluxes translate into line luminosities of $\log L\left(\mathrm{H}_{\alpha}\right)$ / $L_{\odot}=-3.74$ and $\log L\left(\mathrm{H}_{\beta}\right) / L_{\odot}=-5.16$. Using the calibration between line and accretion luminosities proposed by Fang et al. (2009) and Rigliaco et al. (2012), we derive mass accretion rates of $2.9-4.4 \times 10^{-10} M_{\odot} \mathrm{yr}^{-1}$ from $\mathrm{H}_{\alpha}$ and $1.4-4.5 \times 10^{-11} M_{\odot} \mathrm{yr}^{-1}$ from $\mathrm{H}_{\beta}$. If the emission line regions suffer the same amount of reddening as the stellar photosphere, these estimates have to be corrected for extinction. Assuming that the photometric variations are mostly driven by circumstellar extinction, and taking $V \simeq 12.8$ as the reference bright level for the system (cf. Bouvier et al. 1999), we derive $\delta A_{V} \simeq 2.8 \mathrm{mag}$ from the observed $V=15.58$ at the time the spectrum was taken. Correcting for the enhanced extinction, the mass accretion rate becomes $\simeq 7 \times 10^{-9}$ and $\simeq 2 \times 10^{-9} M_{\odot} \mathrm{yr}^{-1}$ from $\mathrm{H}_{\alpha}$ and $\mathrm{H}_{\beta}$, respectively. This range of mass accretion rates is similar to what has been derived for AA Tau in the bright state (e.g. Donati et al. 2010; Valenti et al. 1993). Clearly, the sudden dimming of the system is not associated with a massive accretion outburst or quenching.

The higher resolution XSHOOTER spectrum offers a deeper insight into the shape of the emission line profiles, a few of which are shown in Fig. 5. The $\mathrm{H}_{\alpha}$ and $\mathrm{H}_{\beta}$ line profiles obtained in the deep state are clearly asymmetric and purely in emission. Most noticeably, they lack the deep central absorption seen in similar profiles obtained during the bright state (cf., e.g., Bouvier et al. 2003, Fig. 8; Bouvier et al. 2007, Fig. 8; Donati et al. 2010, Fig. 3). The HeI $1083 \mathrm{~nm}$ line profile has a clear inverse P Cygni signature with a deep redshifted absorption indicative of accretion onto the central object. The profile is qualitatively similar to those reported by Fischer et al. (2008) and obtained in 2002-2006 while AA Tau was still in the bright state. The equivalent width of the HeI redshifted absorption component we measure on the XSHOOTER spectrum is $2.6 \AA$, directly in the center of the range measured for this feature by Fisher et al. (2008). Moreover, the redshifted velocity at which the absorption component reaches back to the continuum is $\sim 320 \mathrm{~km} \mathrm{~s}^{-1}$, similar to that reported in bright state HeI line profiles. Many other lines are seen in emission in the XSHOOTER spectrum, and their equivalent widths are listed in Table 3.

Although we do not have photometric measurements simultaneous to the XSHOOTER spectrum obtained on 25 Feb. 2012 (JD 55983.0270), $V$ - and $R$-band measurements were taken a few nights apart, on 23 and 26 Feb. 2012. Averaging these measurements and combining them to $E W\left(\mathrm{H}_{\alpha}\right)=26 \AA$, we derive from the extinction-corrected $\mathrm{H}_{\alpha}$ line luminosity a mass 
Table 3. Equivalent width of emission lines recorded in the ESO/XSHOOTER spectrum.

\begin{tabular}{lcc|lcc|lcc}
\hline \hline Line & $\begin{array}{c}\lambda \\
(\mathrm{nm})\end{array}$ & $\begin{array}{c}E W \\
\AA\end{array}$ & Line & $\begin{array}{c}\lambda \\
(\mathrm{nm})\end{array}$ & $\begin{array}{c}E W \\
\AA\end{array}$ & Line & $\begin{array}{c}\lambda \\
(\mathrm{nm})\end{array}$ & $\begin{array}{c}E W \\
\AA\end{array}$ \\
\hline $\mathrm{H} 16$ & 370.5 & 2 & $\mathrm{HeI}$ & 447.2 & 1.2 & {$[\mathrm{SII}]$} & 673.1 & 0.17 \\
$\mathrm{H} 15$ & 371.3 & 2.5 & $\mathrm{HeII}$ & 468.7 & 0.4 & $\mathrm{CaII}$ & 849.9 & 0.54 \\
$\mathrm{H} 14$ & 372.2 & 3.0 & $\mathrm{H} \beta$ & 486.1 & 9.0 & $\mathrm{CaII}$ & 854.3 & 0.72 \\
$\mathrm{H} 13$ & 373.5 & 4 & {$[\mathrm{OI}]$} & 557.8 & 0.6 & $\mathrm{CaII}$ & 866.3 & 0.46 \\
$\mathrm{H} 12$ & 375.1 & 7 & {$[\mathrm{SII}]$} & 407.7 & 0.8 & $\mathrm{~Pa} \delta$ & 1004.9 & 1.4 \\
$\mathrm{H} 11$ & 377.1 & 11 & {$[\mathrm{SII}]$} & 406.86 & 4.0 & $\mathrm{HeI}$ & $1083 .(\mathrm{em})$. & 2.0 \\
$\mathrm{H} 10$ & 379.8 & 15 & $\mathrm{HeI}$ & 587.6 & 1.2 & $\mathrm{HeI}$ & $1083 .($ abs. & 2.5 \\
$\mathrm{H} 9$ & 383.6 & 20 & {$[\mathrm{OI}]$} & 630.0 & 3.8 & $\mathrm{~Pa} \gamma$ & 1093. & 1.4 \\
$\mathrm{H} 8$ & 388.9 & 26 & {$[\mathrm{OI}]$} & 636.4 & 0.9 & $\mathrm{~Pa} \beta$ & 1282. & 1.5 \\
$\mathrm{CaIIK}$ & 393.4 & 14.5 & $\mathrm{H} \alpha$ & 656.3 & 26.0 & $\mathrm{H} 2$ & 2122. & 0.3 \\
$\mathrm{CaIIH} / \mathrm{H} \epsilon$ & 397.0 & 31 & {$[\mathrm{NII}]$} & 658.3 & 0.3 & $\mathrm{Br} \gamma$ & 2166. & no emission \\
$\mathrm{H} \delta$ & 410.2 & 13 & $\mathrm{HeI}$ & 667.9 & 0.3 & & & \\
$\mathrm{H} \gamma$ & 434.1 & 14 & $\mathrm{LiI}$ & 670.7 & -0.5 & & & \\
\hline
\end{tabular}
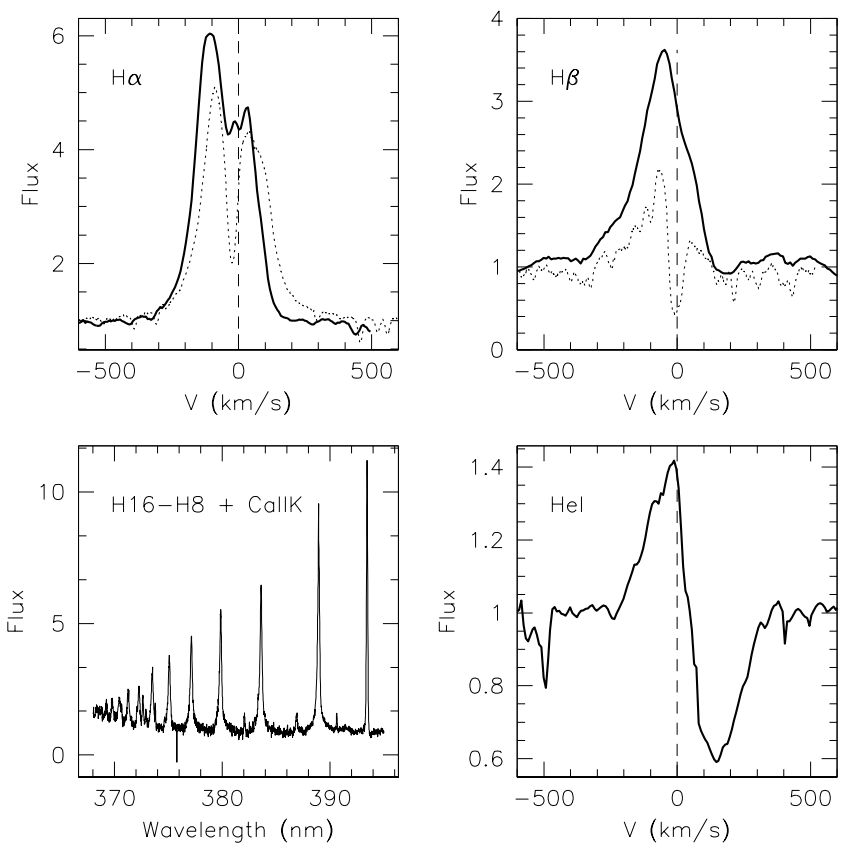

Fig. 5. Line profiles from the mid-resolution VLT/XSHOOTER spectrum obtained on Feb. 25, 2012. From top to bottom and left to right: $\mathrm{H}_{\alpha}, \mathrm{H}_{\beta}$, the higher Balmer series and Ca IIK, and HeI $1083 \mathrm{~nm}$. The lower axis is labeled with velocities corrected for the star's radial velocity $\left(V_{\mathrm{rad}}=17.2 \mathrm{~km} \mathrm{~s}^{-1}\right.$; Donati et al. 2010), except for the higher Balmer series where it is labeled in wavelength. The continuum flux has been scaled to unity for all profiles. The dotted $\mathrm{H}_{\alpha}$ and $\mathrm{H}_{\beta}$ profiles shown in the upper panels are the mean profiles obtained during AA Tau's bright state by Bouvier et al. (2007).

accretion rate of $3.6 \times 10^{-9} M_{\odot} \mathrm{yr}^{-1}$, in agreement with that derived above from the Calar Alto spectrum obtained two months earlier. Hence, we do not find evidence for significant variations of the mass accretion rate on this timescale during the deep state.

The full XSHOOTER spectrum is shown in Fig. 6 and is compared with Kurucz models computed for AA Tau's parameters $\left(T_{\text {eff }}=4000 \mathrm{~K}, R_{*}=1.85 R_{\odot}, d=140 \mathrm{pc}\right.$; from Bouvier et al. 1999). A slightly reddened Kurucz spectrum, with $A_{V}=0.78 \mathrm{mag}$, provides a good match to the bright-state $U B V R I J H K$ photometry of the system. A much higher extinction, $A_{V} \sim 3.78 \mathrm{mag}$, is required to match the red and near-IR regions of the XSHOOTER spectrum obtained in the deep state. This result supports $\mathrm{a} \geq 3$ mag increase of visual extinction on

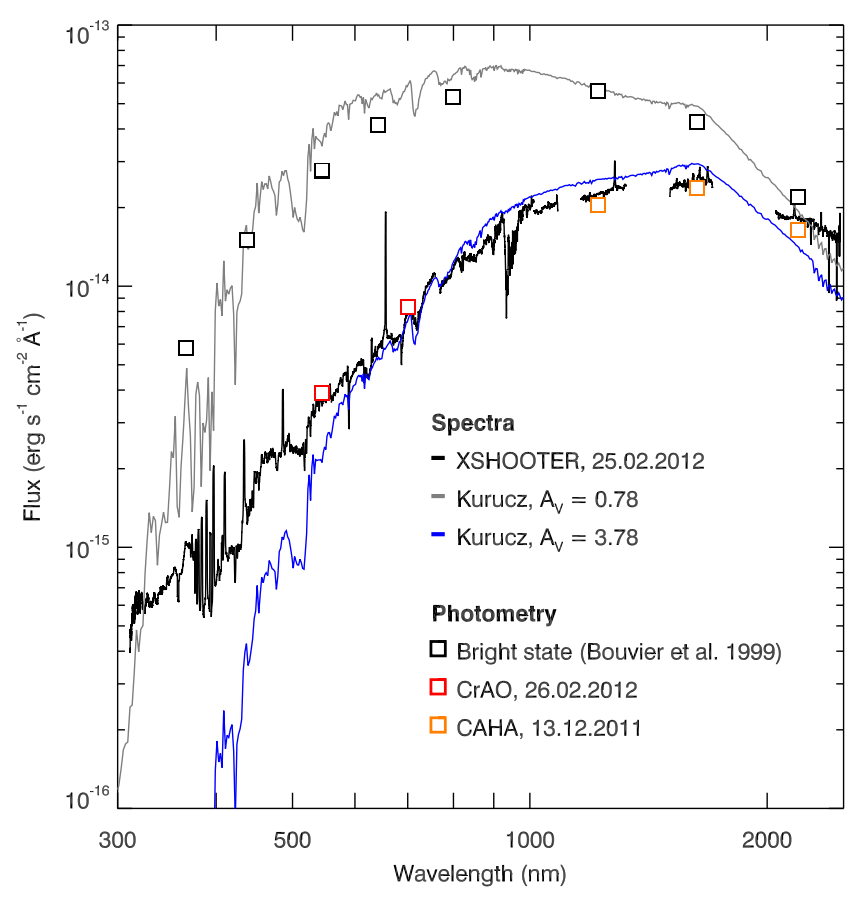

Fig. 6. Flux calibrated VLT/XSHOOTER spectrum obtained on Feb. 25, 2012, is shown (thick black curve) over the 310-2470 $\mathrm{nm}$ wavelength range on a logarithmic scale. Regions affected by strong telluric absorption in the $J H K$ bands are clipped from the spectrum. The red squares corresponds to $V$ and $R_{J}$ photometry obtained at CrAO on Feb. 26, $2012\left(V=14.92, R_{J}=13.30\right)$, and the orange squares to $J H K$ photometry obtained at Calar Alto on Dec. 13, 2011. The upper spectrum (gray curve) is a Kurucz model computed for AA Tau's parameters reported in Bouvier et al. (1999) $\left(T_{\text {eff }}=4000 \mathrm{~K}, R=1.85 R_{\odot}\right.$, $d=140 \mathrm{pc}, A_{V}=0.78 \mathrm{mag}$ ) and the overplotted black squares correspond to the UBVRIJHK bright-state photometry from the same study. The lower spectrum (blue curve) is the same Kurucz model reddened by $A_{V}=3.78 \mathrm{mag}$.

the line of sight as being the cause of AA Tau's sudden deepening. At wavelengths shorter than $700 \mathrm{~nm}$, the system's flux is higher than predicted by the reddened Kurucz model, suggesting that the system's luminosity at shorter wavelengths is dominated by scattered light.

\section{Discussion}

Since it began to be monitored in 1987 at Mount Maidanak Observatory and until 2011, AA Tau has exhibited a relatively 
stable photometric behavior. Yearly light curves exhibit a nearly constant maximum brightness level $(V \simeq 12.3-12.7)$ interrupted by periodic dips with an amplitude up to 1.5 mag occurring every 8.2 days and lasting for a few days (e.g. Artemenko et al. 2012). The $8.2 \mathrm{~d}$ period had originally been reported by Rydgren et al. (1985) from photometry obtained in 1984. The dips have been interpreted has eclipses of the central star by a rotating inner disk warp located at a distance of $8.8 R_{*}$ from the stellar surface (Bouvier et al. 1999; Ménard et al. 2003). The nonaxisymmetric inner disk warp was ascribed to the interaction between the inner disk and an inclined stellar dipole (Bouvier et al. 1999, 2003, 2007). Follow-up spectropolarimetric observations confirmed the strong $(2-3 \mathrm{kG})$ and slightly tilted $\left(\sim 20^{\circ}\right)$ stellar dipole (Donati et al. 2010).

At some point between early and late 2011, AA Tau suddenly faded by $\sim 2$ mag in the $V$ band and remained at around this deep photometric state since then. By comparing the CCD image taken at Calar Alto on Dec. 2011 with the SDSS9 catalog release (Ahn et al. 2012), we verified that the three field stars nearest to AA Tau, located at a projected distance ranging from 26 to 53", did not exhibit any luminosity variation, thus confirming that the sudden dimming is indeed restricted to AA Tau. We additionally constrained the spatial extent of the source of enhanced extinction by investigating whether AA Tau's jet has undergone a similar deepening as the central star. Assuming that the $[\mathrm{OI}] 630.0 \mathrm{~nm}$ line forms in the jet, we computed the line's luminosity for both the bright and the deep photometric states, as $F([\mathrm{OI}]) \simeq F_{R}^{0} \times E W([\mathrm{OI}]) \times 10^{\left(-0.4 \cdot m_{R}\right)}$. For the bright state, we combined the measurement from Hartigan et al. (1995) $E W([\mathrm{OI}])=0.58 \AA$ A obtained on JD 2447169.8 (Jan. 1988) with Maidanak's photometric measurement $R=11.46$ mag secured on JD 2447170.4 , to obtain $F([\mathrm{OI}])=3.2 \times 10^{-14} \mathrm{erg} \mathrm{cm}^{2} \mathrm{~s}^{-1}$. For the deep state, we used our Calar Alto measurement from Dec. 23, 2011, $E W([\mathrm{OI}])=7.5 \AA$ and the simultaneous photometric measurement $R=14.35$, which yields $F([\mathrm{OI}])=$ $2.7 \times 10^{-14} \mathrm{erg} \mathrm{cm}^{2} \mathrm{~s}^{-1}$. We conclude that the jet's luminosity, as measured by the [OI] line flux, has not changed between the bright and deep states. The source of AA Tau's deepening event must therefore be more compact than the jet, at least along the jet direction, which extends to about $21^{\prime \prime}$ at $\mathrm{PA}=183^{\circ}$ from the central star (Cox et al. 2013).

The results presented above and our previous knowledge of the system prompt us to interpret this fading as the consequence of enhanced extinction on the line of sight produced by dust in the disk. Both the near-IR $(J-H, H-K)$ colors of the system in the deep state and the slope of the red and near-IR parts of the XSHOOTER spectrum suggest that the system is suffering an additional 3-4 mag of visual extinction compared with the bright state $\left(A_{V}=0.8 \mathrm{mag}\right.$, Bouvier et al. 1999). The $V$-band magnitude difference between the bright and deep state is, however, only of about 2-3 mag, and the slope of the optical spectra is also bluer than expected for a 4 mag increase of visual extinction. Indeed, the system becomes bluer at optical wavelengths as it reaches minimum brightness (see Fig. 3), a color behavior typical of UX Ori-type variables, whose variations result from obscuration by circumstellar dust (Grinin et al. 1991; Herbst et al. 1994). Altogether, this suggests that, as direct photons are being absorbed by the dusty disk on the line of sight, scattered light largely contributes to, and even dominates the system's optical flux at minimum brightness.

This interpretation provides a consistent framework for understanding not only the photometric properties of the system in the deep state and its spectral slope from UV to near-IR wavelengths, but also accounts for the disappearance of the $8.2 \mathrm{~d}$ periodic eclipses from the optical light curve as well as for the lack of central absorption components in the Balmer line profiles, two features regularly observed in the bright state. Assuming scattered light is a major component of the system's optical flux in the deep state, while direct light is largely suppressed, the photons reaching the observer must have escaped the central system toward unobscured lines of sight, i.e., polewards or at least toward relatively high latitudes. At these angles, reaching well above the disk midplane, the stellar photons do not suffer occultations by the inner disk warp, and therefore do not convey any periodic photometric signal as they are scattered back to the observer. The late recovery of the $8.19 \mathrm{~d}$ period at the end of the 2012/13 season suggests that the scattered/direct light fraction has changed by then. Similarily, the deep central absorption component of the Balmer line profiles, always present in the bright state, presumably arises from the photons being absorbed either very close to the star by the slow-moving material at the top of the accretion funnel flows or farther away at the base of a dense disk wind nearly perpendicular to the line of sight (Kurosawa et al. 2006, 2011). Scattered photons that have escaped the central system along trajectories located well above the disk midplane do not encounter either of these absorbing media and therefore do not exhibit central absorption signatures in the line profiles (cf. Appenzeller et al. 2005; Grinin et al. 2012). Only the HeI $1083 \mathrm{~nm}$ line still displays a pronounced redshifted absorption component in the deep state that is essentially unchanged from the bright state, which is indicative of accretion onto the central star (Fischer et al. 2008). Indeed, at this wavelength scattered light does not dominate any more (cf. Figs. 3 and 6) and the central part of the system, though heavily reddened, can be directly seen.

Because AA Tau is surrounded by a circumstellar disk seen nearly edge-on (Cox et al. 2013), the deep photometric state the system currently undergoes most likely results from a drastic change in the disk geometry, at least on the line of sight. What is the physical process at the origin of the enhanced extinction on the line of sight, and at which distance in the disk is the obscuring material located?

Star-disk interaction MHD models indicate that various kinds of instabilities may occur at the inner disk edge. For instance, Romanova et al. (2013) have shown that one-arm bending waves can develop as the inner disk responds to an inclined stellar dipole, which results in an inner disk warp whose scale height $h$ may exceed $0.3 r$. Such a warp is held responsible for AA Tau's eclipses in the bright state (Bouvier et al. 1999) and an average warp scale-height of $h / r \sim 0.3$ has been deduced from the statistical analysis of an ensemble of AA Tau-like light curves in NGC 2264 (Alencar et al. 2010). The warp geometry is sensitive to the topology and inclination of the stellar magnetic field and to the mass accretion rate in the inner disk (Romanova et al. 2012). While the deepening of the AA Tau system could be tentatively ascribed to a sudden increase of the warp scale height, resulting in the total occultation of the central star, we consider this interpretation unlikely. Firstly, any change in the inner disk property would probably affect the mass accretion rate onto the star. Yet, our estimates of mass accretion rate obtained in the deep state are not significantly different from those measured in the bright state. Second, the near-IR colors of the system in the deep state are reddened by at least 4 mag of visual extinction. The near-IR flux comes from the inner disk regions, which suggests that the obscuring material is located beyond a few $0.1 \mathrm{AU}$ from the central star, i.e., much farther away than the inner disk edge ( $r \sim 0.08$ AU; Bouvier et al. 1999). Third, AA Tau's light 
curve in the last season shows a slowly varying extinction pattern with little photometric dispersion that occurs on a timescale of $\sim 100$ days. If this timescale is to be related to Keplerian rotation in the disk, it points to radial distances well beyond the inner edge. More generally, the extended duration of the deepening episode ( $\geq 2 \mathrm{yr}$ ) seems to exclude any short-timescale instability operating in the inner disk, which would be expected to produce transient variations of the disk scale height (e.g., Turner et al. 2010).

Several lines of evidence thus point to the obscuring material being located far beyond the inner disk edge. The sudden appearance of additional extinction on the line of sight could conceivably result from the growth of an instability that acts to increase the disk scale-height on long timescales (e.g. Simon et al. 2011; Flock et al. 2012; Lesur et al. 2013). This process remains speculative because detailed physical conditions in the disk, such as viscosity, magnetic field, etc., are unknown. However, we note that these unstabilities may eventually trigger FU Ori outbursts as they propagate inward (Zhu et al. 2010; Bae et al. 2013), thus potentially making AA Tau a pre-FUOr candidate. Disk instabilities may also trigger dense disk winds (e.g., Lesur et al. 2013; Bai \& Stone 2013) that may be the source of enhanced lineof-sight extinction if they are able to lift up dust from the disk mid-plane (Owen et al. 2011; Bans \& Königl 2012). However, AA Tau's HeI $1083 \mathrm{~nm}$ line profile does not bear evidence for significant wind absorption. Alternatively, enhanced extinction could also result from an azimuthal disk asymmetry coming onto the line of sight at Keplerian velocity. Nonaxisymmetric density and associated scale-height bumps may be triggered in the disk by an embedded planetary mass companion (e.g., Uribe et al. 2011; Kley \& Nelson 2012; Baruteau \& Masset 2013) or arise from instability driven density vortices (e.g., Lesur \& Papaloizou 2010; Meheut et al. 2012; Lin 2012). Assuming the disk overdensity rotates at Keplerian velocity, the observed timescales for AA Tau's photometric variations provide some constraints on its radial distance from the central star. Because the bright state prior to the deepening episode lasted for at least 24 years, the orbital period of the disk perturbation must be at least this long. Assuming Keplerian rotation around the central $0.8 M_{\odot}$ star, this locates the density bump at a distance of $7.7 \mathrm{AU}$ or more from the central star. At this distance, the $2 \mathrm{yr}$ duration of the on-going deep state implies an azimuthal extent of at least 30 degrees in the disk. Shorter timescale photometric variations observed within the deep state, ranging from days to months (cf. Fig. 1), furthermore suggest that the azimuthal distribution of dust in the density perturbation is not homogeneous.

To investigate whether AA Tau could have been in such a faint state in previous times, we searched all available publications providing AA Tau's photometry up to the mid-80s, i.e., before the yearly Maidanak monitoring project started, based on the bibliography linked to the object at CDS Strasbourg. Joy (1949) first reported $V \simeq 14$, with the warning that "magnitude estimates are rough aproximations" because they were derived from low-resolution photographic spectra. The oldest actual photometric measurement we found was reported by Varsavsky (1960), who listed $V=12.82$ obtained in late 1959. A few other measurements from the early 60s are listed in Smak (1964), who reported $V=11.9-12.9$ for three observations obtained in 1962, suggesting that the system was already in a bright state at that epoch. Nurmanova (1983) reported $20 \mathrm{~V}$-band observations of the system spanning from 1974 to 1979 , yielding $V=12.5-14.2 \mathrm{mag}$. Indeed, all published $V$-band values we could find in the literature from the 60 s to the mid- 80 s lie in the range from about 12.4 to $14.2 \mathrm{mag}$, i.e., similar to the photometric range of the long-term Maidanak light-curve covering from 1987 onward. A large number of fainter measurements are reported in the literature, but these correspond to photographic magnitudes, i.e., closer to the $B$ band. We thus did not find any compelling evidence for a prior deepening episode of the system since the late 50s. However, we cannot totally exclude it either, since until the yearly monitoring started in 1987 at Maidanak Observatory the system has been only sporadically observed and the duration of the deepening events is unknown as yet.

\section{Conclusion}

While the young, low-mass AA Tau accreting system has exhibited a stable photometric behavior during a period extending over 24 years (1987-2011), we have reported here its sudden dimming by about 2 mag that started in 2011 and is still on-going. The photometric and spectral properties we derived for the system in the deep state indicate that it currently suffers $\geq 4$ mag of visual extinction on the line of sight and is dominated by scattered light at optical wavelengths. The mass accretion rate onto the star and in the inner disk has not significantly changed between the previous 24 yr-long bright state and the recent dimming episode. We proposed that the additional amount of extinction on the line of sight is produced by a density perturbation in the disk located at a distance of $\geq 7.7$ AU from the central star. This density perturbation might result from a lowmass companion embedded in the disk that orbits the central star at Keplerian velocity, possibly a planetary embryo.

Acknowledgements. It is a pleasure to thank Paula Teixeira for help in preparing the XSHOOTER observations, Svetlana Artemenko for assistance during CrAO observations, Geoffroy Lesur and Christophe Pinte for discussions about disk instabilities and radiative transfer, respectively. We thank the referee for a prompt and useful report. This research has made use of the SIMBAD database, operated at CDS, Strasbourg, France. We acknowledge partial funding from CNRS DERCI on a French-Ukrainian collaborative program, as well as funding from Université Joseph Fourier Grenoble 1 for K. Grankin's stay at IPAG. This study was supported in part by the grant ANR 2011 Blanc SIMI5-6 02001 "Toupies: Towards understanding the spin evolution of stars" (http://ipag.osug.fr/Anr_Toupies/). We acknowledge financial support from CNRS-INSU's Programme National de Physique Stellaire.

\section{References}

Ahn, C. P., Alexandroff, R., Allende Prieto, C., et al. 2012, ApJS, 203, 21 Alencar, S. H. P., Teixeira, P. S., Guimarães, M. M., et al. 2010, A\&A, 519, A88 Appenzeller, I., Bertout, C., \& Stahl, O. 2005, A\&A, 434, 1005

Artemenko, S. A., Grankin, K. N., \& Petrov, P. P. 2012, Astron. Lett., 38, 783 Bae, J., Hartmann, L., Zhu, Z., \& Gammie, C. 2013, ApJ, 764, 141

Bai, X.-N., \& Stone, J. M. 2013, ApJ, 769, 76

Bans, A., \& Königl, A. 2012, ApJ, 758, 100

Baruteau, C., \& Masset, F. 2013, Lect. Not. Phys., 861, 201

Bertout, C., Basri, G., \& Bouvier, J. 1988, ApJ, 330, 350

Bouvier, J., Chelli, A., Allain, S., et al. 1999, A\&A, 349, 619

Bouvier, J., Grankin, K. N., Alencar, S. H. P., et al. 2003, A\&A, 409, 169

Bouvier, J., Alencar, S. H. P., Harries, T. J., Johns-Krull, C. M., \& Romanova, M. M. 2007a, Protostars and Planets V, eds. B. Reipurth, D. Jewitt, \& K. Keil, 479

Bouvier, J., Alencar, S. H. P., Boutelier, T., et al. 2007b, A\&A, 463, 1017

Camenzind, M. 1990, Rev. Mod. Astron., 3, 234

Cardelli, J. A., Clayton, G. C., \& Mathis, J. S. 1989, ApJ, 345, 245

Cody, A. M., Tayar, J., Hillenbrand, L. A., Matthews, J. M., \& Kallinger, T. 2013, AJ, 145,79

Cox, A. W., Grady, C. A., Hammel, H. B., et al. 2013, ApJ, 762, 40

D’Odorico, S., Dekker, H., Mazzoleni, R., et al. 2006, Proc. SPIE, 6269

Donati, J.-F., \& Landstreet, J. D. 2009, ARA\&A, 47, 333

Donati, J.-F., Skelly, M. B., Bouvier, J., et al. 2010, MNRAS, 409, 1347

Fang, M., van Boekel, R., Wang, W., et al. 2009, A\&A, 504, 461 
J. Bouvier et al.: AA Tauri's sudden and long-lasting deepening: enhanced extinction by its circumstellar disk

Fischer, W., Kwan, J., Edwards, S., \& Hillenbrand, L. 2008, ApJ, 687, 1117 Flock, M., Dzyurkevich, N., Klahr, H., Turner, N., \& Henning, T. 2012, ApJ, 744,144

Grankin, K. N., Melnikov, S. Y., Bouvier, J., Herbst, W., \& Shevchenko, V. S. 2007, A\&A, 461, 183

Grinin, V. P., Kiselev, N. N., Chernova, G. P., Minikulov, N. K., \& Voshchinnikov, N. V. 1991, Ap\&SS, 186, 283

Grinin, V. P., Tambovtseva, L. V., \& Weigelt, G. 2012, A\&A, 544, A45

Hartigan, P., Edwards, S., \& Ghandour, L. 1995, ApJ, 452, 736

Herbig, G. H., \& Kameswara Rao, N. 1972, ApJ, 174, 401

Herbst, W., Herbst, D. K., Grossman, E. J., \& Weinstein, D. 1994, AJ, 108, 1906

Joy, A. H. 1949, ApJ, 110, 424

Kenyon, S. J., \& Hartmann, L. 1995, ApJS, 101, 117

Kley, W., \& Nelson, R. P. 2012, ARA\&A, 50, 211

Koenigl, A. 1991, ApJ, 370, L39

Kovács, Z., Mall, U., Bizenberger, P., Baumeister, H., \& Röser, H.-J. 2004, Proc. SPIE, 5499, 432

Kuhi, L. V. 1974, A\&AS, 15, 47

Kurosawa, R., Harries, T. J., \& Symington, N. H. 2006, MNRAS, 370, 580

Kurosawa, R., Romanova, M. M., \& Harries, T. J. 2011, MNRAS, 416, 2623

Lesur, G., \& Papaloizou, J. C. B. 2010, A\&A, 513, A60

Lesur, G., Ferreira, J., \& Ogilvie, G. I. 2013, A\&A, 550, A61

Lin, M.-K. 2012, ApJ, 754, 21

Meheut, H., Keppens, R., Casse, F., \& Benz, W. 2012, A\&A, 542, A9
Meisenheimer, K. 1994, Sterne und Weltraum, 33, 516

Ménard, F., Bouvier, J., Dougados, C., Mel'nikov, S. Y., \& Grankin, K. N. 2003, A\&A, 409, 163

Modigliani, A., Goldoni, P., Royer, F., et al. 2010, Proc. SPIE, 7737

Nurmanova, U. A. 1983, Peremennye Zvezdy, 21, 777

O'Sullivan, M., Truss, M., Walker, C., et al. 2005, MNRAS, 358, 632

Owen, J. E., Ercolano, B., \& Clarke, C. J. 2011, MNRAS, 411, 1104

Rigliaco, E., Natta, A., Testi, L., et al. 2012, A\&A, 548, A56

Romanova, M. M., Ustyugova, G. V., Koldoba, A. V., \& Lovelace, R. V. E. 2012, MNRAS, 421, 63

Romanova, M. M., Ustyugova, G. V., Koldoba, A. V., \& Lovelace, R. V. E. 2013 , MNRAS, 430, 699

Rydgren, A. E., Vrba, F. J., Chugainov, P. F., \& Shakhovskaya, N. I. 1985, BAAS, 17, 556

Simon, J. B., Hawley, J. F., \& Beckwith, K. 2011, ApJ, 730, 94

Smak, J. 1964, ApJ, 139, 1095

Turner, N. J., Carballido, A., \& Sano, T. 2010, ApJ, 708, 188

Valenti, J. A., Basri, G., \& Johns, C. M. 1993, AJ, 106, 2024

Vandame, B. 2002, Proc. SPIE, 4847, 123

Vernet, J., Dekker, H., D’Odorico, S., et al. 2011, A\&A, 536, A105

Terquem, C., \& Papaloizou, J. C. B. 2000, A\&A, 360, 1031

Uribe, A. L., Klahr, H., Flock, M., \& Henning, T. 2011, ApJ, 736, 85

Varsavsky, C. M. 1960, ApJ, 132, 354

Zhu, Z., Hartmann, L., Gammie, C. F., et al. 2010, ApJ, 713, 1134 